\title{
Prevalence of nutritional risk and its impact on functional recovery in older inpatients on maintenance hemodialysis: a retrospective single-center cohort study
}

\author{
Yoshitaka Shimizu*, Tatsu Fujiura and Hidetaka Wakabayashi
}

\begin{abstract}
Background: Poor nutritional status and functional impairment are common in patients with end-stage renal disease (ERSD) on maintenance hemodialysis (MHD). Although nutritional status is associated with functional dependence and rehabilitation outcome in several diseases, this association remains unclear in patients with ESRD. The aim of this study was to investigate nutritional risk and its impact on rehabilitation outcomes in MHD inpatients who required rehabilitation.
\end{abstract}

Methods: A retrospective cohort study was performed in 57 consecutive MHD inpatients aged 65 or older who had undergone rehabilitation. The Geriatric Nutritional Risk Index (GNRI) was used to assess nutritional risk and was calculated from height, dry body weight, and serum albumin level at the start of rehabilitation. Nutritional risk was defined as a GNRI < 91.2. The activities of daily living were used as a measure of rehabilitation outcome and were assessed by the Barthel Index (BI) at the start of rehabilitation and discharge. The Mann-Whitney $U$ test and multiple regression analysis were performed. In the multiple regression analysis, BI gain was the dependent variable and age, sex, and GNRI were the independent variables.

Results: The study included 34 men and 23 women. Mean ( \pm SD) GNRI was $79.8 \pm 9.9$. Of the 57 patients, 50 (87.7\%) were identified as having a nutritional risk and 7 were not. The gain in BI was significantly higher in patients without nutritional risk (median 50 vs. 10, $p=0.03$ ). Multiple regression analysis showed GNRI was associated independently with $\mathrm{Bl}$ gain $\left(R^{2}=0.14, \beta=0.29, p=0.03\right)$.

Conclusions: The majority of the MHD patients who underwent rehabilitation had a nutritional risk. Nutritional risk was associated independently with functional recovery.

Keywords: Hemodialysis, Geriatric Nutritional Risk Index, Activities of daily living, Rehabilitation

\section{Background}

Poor nutritional status and functional impairment are common in patients with end-stage renal disease (ESRD) on maintenance hemodialysis (MHD). In chronic kidney disease, poor nutritional status due to multiple factors is categorized as protein-energy wasting and is associated with adverse clinical outcomes especially in ERSD patients [1]. The reported prevalence of malnutrition in dialysis patients ranges between 18 and 75\% [2, 3]. In addition,

\footnotetext{
* Correspondence: shimizu198966@gmail.com

Department of Rehabilitation Medicine, Yokohama City University Medical Center, 4-57 Urafune-chou, Minami ward, Yokohama City 232-0024, Japan
}

19-26\% of MHD patients have a functional impairment in activities of daily living (ADLs); that is, they cannot perform one or more tasks in ADLs without assistance such as bathing, dressing, toilet use, transfer, feeding, and continence $[4,5]$. This is especially apparent in MHD patients aged 65 years or older, with $52 \%$ reported to have impaired ADLs [6]. Because ADL impairment is associated with mortality in MHD patients [4], it is important to prevent and minimize these impairments. Rehabilitation is one method for treating impaired ADLs, and there are some ADL measures that can be used to assess rehabilitation outcomes [7, 8]. Although 
rehabilitation is very important, there have only been a small number of published reports on rehabilitation outcomes in MHD patients with impaired ADLs [9].

Although nutritional status is associated with functional dependence and rehabilitation outcome in several diseases, this association remains unclear in patients with ESRD. A meta-analysis of 240 studies showed malnutrition, and related risks were associated directly with functional dependency [10]. It has also been reported that malnutrition is associated with poor rehabilitation outcomes in patients with stroke [11], chronic heart failure [12], or chronic obstructive pulmonary disease [12]. However, we are unaware of any previous reports on the association between nutritional status and rehabilitation outcomes in MHD patients.

The aim of this study was therefore to assess nutritional risk and its impact on rehabilitation outcomes in MHD inpatients who required rehabilitation.

\section{Methods}

A retrospective cohort study was performed in 57 inpatients who had been admitted consecutively to the Yokohama City University Medical Center and were subsequently referred by attending physicians to the Department of Rehabilitation Medicine between April 2015 and March 2017.

The study included patients aged 65 or older who had undergone both maintenance hemodialysis and rehabilitation during their hospital stay. Patients were excluded from the study if they had undergone new or temporary hemodialysis, had less than two rehabilitation sessions, or had died during their hospital stay.

All participants underwent hemodialysis three times a week and physical therapy five times a week. They underwent physical therapy of 20 or 40 min per session, while some participants received occupational therapy and/or speech therapy if required. Rehabilitation was performed mainly in an exercise room but sometimes was carried out at the bedside when patients could not come to the exercise room because of their physical condition. According to the disease, general condition, and ADLs of each patient, the doctors made a rehabilitation order that included the location, outline of the program, frequency, and length of each session. The therapist in charge of each patient decided the details of the rehabilitation program. Each program was designed with the purpose of improving or maintaining ADL. Each program mainly included a range of motion exercises, resistance training, and practice of ADLs. The practice of ADLs regarding moving such as sitting, standing up, walking, and going up and down stairs was performed as physical therapy. The practice of other activities such as feeding, grooming, and dressing was performed as occupational therapy and speech therapy. If the patient was fully independent for ADLs, resistance training and aerobic exercise were performed to achieve further improvement in physical function.
Nutritional status was assessed by the Geriatric Nutritional Risk Index (GNRI) [13]. This index has been reported to be an accurate tool for identifying MHD patients at nutritional risk [14] and is calculated as GNRI $=\{14.89 \times$ serum albumin $(\mathrm{g} / \mathrm{dl})\}+\{41.7 \times($ body weight/ideal body weight)\}. The ideal body weight was defined as the value calculated from height and a body mass index (BMI) of $22 \mathrm{~kg} / \mathrm{m}^{2}$. In cases with a (body weight/ideal body weight) $>1$, this value was replaced by 1 [13]. For this study, GNRI was calculated from the serum albumin level and body weight measured at the hemodialysis session closest to the start of rehabilitation. Serum albumin level (g/dL) was measured by an improved bromocresol purple (BCP) method before hemodialysis, body weight $(\mathrm{kg})$ after hemodialysis, and height $(\mathrm{cm})$ at admission, with the values expressed to one decimal place. In accordance with a previous study [14], the risk of malnutrition in MHD patients was defined as a GNRI $<91.2$.

ADLs were evaluated by physical therapists in charge of each patient at the start of rehabilitation and at discharge using the Barthel Index (BI). The BI consists of the following ten items: feeding, moving from bed to a wheelchair and back, grooming, toilet use, bathing, mobilization (level surface), going up and down stairs, dressing, bowel continence, and bladder continence. The BI score ranges from 0 to 100 , with higher scores indicating greater functional independence of ADLs [8]. Age, sex, C-reactive protein (CRP, $\mathrm{mg} / \mathrm{dL}$ ), etiology of chronic kidney disease, causative disease for admission, and number of days from admission to the start of rehabilitation and from the start of rehabilitation to discharge were also recorded.

A previous study demonstrated that the minimal clinically important difference of the 20-point BI in stroke patients was 1.85 [15], equivalent to 9.25 in the 100 -point BI scale. In patients with chronic kidney disease who were hospitalized and underwent physical therapy, the standard deviation of the BI was reported to be 13.4 [16]. Assuming the proportion of patients with or without nutritional risk was equal and using the values $\alpha=0.05, \beta=0.8, \delta=9.25$, and $\sigma=13.4$, we calculated that it was necessary to include 34 participants in each group in order to reject the null hypothesis that the gain in BI was equal. We therefore planned to recruit 68 patients in the study.

The statistical analyses were performed using JMP ${ }^{\bullet}$ Pro 12.2 (SAS Institute Inc.; Cary, NC, USA). Fisher's exact test and the Mann-Whitney $U$ test were used to determine whether or not there were differences in the characteristics of patients with or without a risk of malnutrition. A multiple regression analysis was performed to examine whether GNRI was associated independently with the gain in BI from the start of rehabilitation to discharge. In this analysis, the dependent variable was BI gain, and the independent variables were age, sex, and GNRI. Age and sex were selected as the regression factors because according 
to previous studies $[17,18]$, they have a strong probability of affecting rehabilitation outcome. A $p$ value $<0.05$ was considered statistically significant.

\section{Results}

A total of 100 patients underwent both hemodialysis and rehabilitation during the study period. Thirty-seven patients who underwent new or temporary hemodialysis during their hospital stay, 1 patient who underwent rehabilitation less than two times, and 5 patients who died were excluded from the analysis. The remaining 57 patients ( 34 men, 23 women) were included in the study; all underwent physical therapy, 14 had occupational therapy, and 13 received speech therapy.

Table 1 shows the results of the comparison of differences in clinical indices between patients with or without a risk of malnutrition. Mean GNRI $( \pm \mathrm{SD})$ at the start of rehabilitation was $79.8 \pm 9.9$. Fifty patients $(87.7 \%)$ were identified as being at risk of malnutrition and 7 patients (12.3\%, all males) were not. Median BI at the start of rehabilitation was 35 with an interquartile range of 10-60. The gain in BI from the start of rehabilitation to discharge was significantly greater in patients not at risk of malnutrition than in patients at risk of malnutrition. There was no significant difference between the two groups in the number of days from either admission to the start of rehabilitation or the start of rehabilitation to discharge.

Table 2 shows the results of the multiple regression analysis of the gain in BI adjusted for age, sex, and GNRI. GNRI was the only factor associated independently with BI gain $(\beta=0.268,95 \%$ confidence intervals 0.080 , $1.494, p=0.030)$. We estimated that CRP may affect serum albumin concentration. However, CRP could not be included in the multiple regression analysis because it correlated strongly with both serum albumin concentration $(r=-0.607, p<0.001)$ and GNRI $(r=-0.523, p<0.001)$.

\section{Discussion}

This study obtained two main findings regarding the prevalence of nutritional risk and its impact on rehabilitation outcome in older MHD inpatients who had undergone rehabilitation. Firstly, $87.7 \%$ of MHD patients who underwent rehabilitation were identified as having a nutritional risk, assessed by GNRI. Secondly, nutritional risk was associated independently with rehabilitation outcome.

The majority of MHD patients in our study who underwent rehabilitation were identified as having a nutritional risk. Yamada et al. reported a prevalence of 39.3\% for nutritional risk in MHD patients [14], a value lower than that observed in our study. A possible reason for this difference may be variations in the characteristics of the subjects, as the Yamada et al. study was on outpatients and also included patients younger than 65 years. Evidence from a meta-analysis of 240 studies, including various healthcare settings, showed malnutrition, and its risks were associated directly with a setting-related level of dependence. The prevalence of malnutrition in rehabilitation/sub-acute care was also shown to be higher than in all other settings including the community, outpatients, home-care services, hospitals, nursing homes, and longterm care [10]. Granger et al. reported that a BI of 60 points appeared to be a pivotal score at which stroke patients moved from functional dependency to assisted independence [19]. In the present study, the upper quartile of BI at the start of rehabilitation was 60 , and therefore, the majority of the patients were considered to be functionally dependent. It is possible this high rate of patients with functional dependency was the reason for the high prevalence of nutritional risk observed in our study. We therefore consider that nutritional assessment is very important in MHD patients who are undergoing rehabilitation.

Our data showed nutritional risk in MHD inpatients was associated independently with a gain in the BI and that patients without nutritional risk achieved greater gains in this index. Other studies have reported the impact of GNRI score in MHD patients on mortality [20], physical function [21], and quality of life [21]. Our study is the first to show an influence of GNRI on rehabilitation outcome in MHD patients. This result indicates that the GNRI may be useful for predicting rehabilitation outcomes in MHD inpatients. We consider a cutoff value of GNRI $<91.2$ may be useful for predicting whether or not rehabilitation will be more effective.

The high prevalence of nutritional risk and its impact on rehabilitation outcomes indicate the importance of rehabilitation nutrition. Yoshimura et al. reported that nutritional intervention added to resistance training during convalescent rehabilitation improved ADLs in older patients with a decreased muscle mass [22]. There are only a small number of reports on the combination of nutritional intervention and exercise training in adult MHD patients, and the benefit of this combination therefore remains uncertain $[23,24]$. In addition, there are no reports on this combination in older MHD patients or MHD patients with impaired ADLs. Nutritional intervention added to rehabilitation has the potential to improve both nutritional status and rehabilitation outcomes in MHD patients with impaired ADLs. There is also evidence in patients with stroke and hip fracture that nutritional improvement is associated with greater gains in ADLs [25-27]. The concept of rehabilitation nutrition involving rehabilitation and management of nutritional care is important in MHD patients with impaired ADLs [28].

This study had some limitations. First, the only subjects in the study were those referred to the Department of Rehabilitation Medicine and did not include patients admitted consecutively to the target hospital during the 
Table 1 Characteristics and comparison of clinical indices in patients with or without a nutritional risk

\begin{tabular}{lllll}
\hline & Total $(n=57)$ & With a nutritional risk $(n=50)$ & Without a nutritional risk $(n=7)$ & $P$ value \\
\hline Age (years), mean \pm SD & $73.8 \pm 6.4$ & $73.8 \pm 6.5$ & $70.5 \pm 5.8$ & $7 / 0$ \\
Sex, males/females & $34 / 23$ & $27 / 23$ & $3.65 \pm 0.2$ \\
Albumin (g/dL), mean \pm SD & $2.8 \pm 0.6$ & $2.7 \pm 0.4$ & $22.1 \pm 2.4$ \\
BMI (kg/m²), mean \pm SD & $20.8 \pm 3.1$ & $20.6 \pm 3.2$ & $96.0 \pm 1.9$ \\
GNRI, mean \pm SD & $79.8 \pm 9.9$ & $77.6 \pm 8.3$ & $0.441(0.235-1.863)$ \\
CRP (mg/dL), median (IQR) & $3.365(0.98-7.742)$ & $3.797(1.621-9.847)$ & $30(7.5-45)$ \\
Bl at baseline, median (IQR) & $35(10-60)$ & $35(11.25-60)$ & $85(77.5-92.5)$ \\
Bl at discharge, median (IQR) & $70(30-90)$ & $65(25-90)$ & $50(25-85)$ \\
Bl gain, median (IQR) & $15(0-30)$ & $10(0-30)$ & $29(19.5-44)$ \\
Length of stay (days), median (IQR) & $37(22-52)$ & $38.5(23.25-51.25)$ & $7(3-8.5)$ & 0.001 \\
Days from A to R (days), median (IQR) & $8(5-14)$ & $9(6-14)$ & $25(15-35.5)$ & 0.016
\end{tabular}

Causative disease for admission ( $n$ )

\begin{tabular}{|c|c|c|}
\hline Ischemic heart disease & 10 & 9 \\
\hline Aortic disease & 4 & 4 \\
\hline Valvular disease of the heart & 4 & 4 \\
\hline Congestive heart failure & 3 & 3 \\
\hline Cerebral infarction & 7 & 3 \\
\hline Parkinson's disease & 1 & \\
\hline Lumbar disease & 6 & 6 \\
\hline Fracture & 3 & 3 \\
\hline Osteoarthritis & 3 & 3 \\
\hline Psychiatric disease & 3 & 2 \\
\hline Perforation of the digestive tract & 2 & 2 \\
\hline Pneumonia & 2 & 2 \\
\hline Shunt complication & 2 & 2 \\
\hline Other & 7 & 7 \\
\hline \multicolumn{3}{|l|}{ tiology of chronic kidney disease $(n)$} \\
\hline Diabetic nephropathy & 30 & 27 \\
\hline Nephrosclerosis & 9 & 6 \\
\hline Chronic glomerulonephritis; & 6 & 6 \\
\hline Multiple renal cysts & 3 & 2 \\
\hline Rapidly progressive glomerulonephritis & 1 & 1 \\
\hline SLE nephritis & 1 & 1 \\
\hline Nephronophthisis & 1 & 1 \\
\hline Chronic pyelonephritis & 1 & 1 \\
\hline Drug-induced kidney injury & 1 & 1 \\
\hline Renal infarction & 1 & 1 \\
\hline Unknown & 3 & 3 \\
\hline
\end{tabular}

1 3 3

$B M I$ body mass index, GNRI Geriatric Nutritional Risk Index, $B I$ Barthel Index, Days from $A$ to $R$ days from admission to start of rehabilitation, IQR interquartile range, SLE systemic lupus erythematosus 
Table 2 Results of the multiple regression analysis with gain in the Barthel Index as the dependent variable

\begin{tabular}{lllll}
\hline & $B$ & $\beta$ & $95 \%$ confidence interval of $B$ & $P$ value \\
\hline Age & -0.997 & -0.234 & $-2.098,0.1046$ & 0.075 \\
Sex & 2.686 & 0.098 & $-4.421,9.794$ & 0.452 \\
GNRI & 0.787 & 0.268 & $0.080,1.494$ & 0.030 \\
\hline
\end{tabular}

$R^{2}=0.139$

GNRI Geriatric Nutritional Risk Index

study period. The results of this study can therefore not be applied to the general population of hospitalized MHD patients. Second, the nutritional risk was assessed using only the GNRI and did not include other more reliable and detailed measures. Third, the requisite sample size was not reached. Fourth, we used serum albumin level measured by an improved BCP method that has been used widely over the past decade, replacing the bromocresol green (BCG) method. It has been reported that the percentage of malnourished patients assessed by controlling nutritional status (CONNUT) differs depending on whether the BCP or BCG method is used to measure albumen level [29]. The Japanese Society of Laboratory Medicine recommends that if the serum albumin level measured by the BCP method is $\leq 3.5 \mathrm{~g} / \mathrm{L}$, the cutoff value should be modified from the value developed for the BCG method [30]. Because the BCG method was the main test used in the past, the GNRI would have been developed using the serum albumin level measured by that method. Therefore, it is possible that the serum albumin level needs to be modified in our study. Furthermore, we were not able to consider factors in the multiple regression analysis that were likely to affect the gain in BI, such as causative disease for admission because of the small number of the participants, and CRP because of its multicollinearity with the GNRI. Further studies investigating the association between nutritional status and rehabilitation outcome in MHD patients should perform a more detailed nutritional assessment in order to diagnose malnutrition and protein-energy wasting.

\section{Conclusion}

The majority of the MHD patients who underwent rehabilitation had a nutritional risk. Nutritional risk was associated independently with rehabilitation outcome. The GNRI has potential as a predictor of rehabilitation outcome in MHD patients.

\section{Abbreviations}

ADLs: Activities of daily living; Bl: Barthel Index; BMl: Body mass index; ERSD: End-stage renal disease; GNRI: Geriatric Nutritional Risk Index; MHD: Maintenance hemodialysis

\section{Acknowledgements}

We are grateful to all the staff in the Department of Rehabilitation Medicine, Yokohama University Medical Center.

\section{Funding}

We have no financial support.
Availability of data and materials

All data generated or analyzed during this study are included in this article.

\section{Authors' contributions \\ YS conceived the study and drafted the manuscript. YS, TF, and HW interpreted the data and revised the manuscript. All authors read and approved the final manuscript.}

\section{Ethics approval and consent to participate}

This study was approved by the ethics committee of the Yokohama City University Medical Center. The approved number is B171100008. The study protocol and contact address/telephone number were available on the website of the Yokohama City University Medical Center, so all participants had the opportunity to decline to participate in the study.

\section{Consent for publication}

Not applicable.

\section{Competing interests}

The authors declare that they have no competing interests.

\section{Publisher's Note}

Springer Nature remains neutral with regard to jurisdictional claims in published maps and institutional affiliations.

Received: 21 July 2018 Accepted: 8 November 2018

Published online: 19 November 2018

\section{References}

1. Fouque D, Kalantar-Zadeh K, Kopple J, Cano N, Chauveau P, Cuppari L, et al. A proposed nomenclature and diagnostic criteria for protein-energy wasting in acute and chronic kidney disease. Kidney Int. 2008;73(3):391-8.

2. Kopple JD. McCollum Award Lecture, 1996: protein-energy malnutrition in maintenance dialysis patients. Am J Clin Nutr. 1997;65(5):1544-57.

3. Kalantar-Zadeh K, Ikizler TA, Block G, Avram MM, Kopple JD. Malnutritioninflammation complex syndrome in dialysis patients: causes and consequences. Am J Kidney Dis. 2003;42(5):864-81.

4. Bossola M, Di Stasio E, Antocicco M, Pepe G, Tazza L, Zuccalà G, et al. Functional impairment is associated with an increased risk of mortality in patients on chronic hemodialysis. BMC Nephrol. 2016;17(1):72. https://doi. org/10.1186/s12882-016-0302-y.

5. Jassal SV, Karaboyas A, Comment LA, Bieber BA, Morgenstern H, Sen A, et al. Functional dependence and mortality in the international dialysis outcomes and practice patterns study (DOPPS). Am J Kidney Dis. 2016;67(2):283-92.

6. Cook WL, Jassal SV. Functional dependencies among the elderly on hemodialysis. Kidney Int. 2008;73(11):1289-95.

7. Katz S, Ford Ford AB, Moskowitz RW, Jackson BA, Jaffe MW. Studies of illness in the aged. The index of ADL: a standardized measure of biological and psychosocial function. JAMA. 1963;185:914-9.

8. Mahoney FI, Barthel DW. Functional evaluation: the Barthel Index. Md State Med J. 1965;14:61-5

9. Endo M, Nakamura $Y$, Murakami T, Tsukahara $H$, Watanabe $Y$, et al. Rehabilitation improves prognosis and activities of daily living in hemodialysis patients with low activities of daily living. Phys Ther Res. 2017; 20(1):9-15.

10. Cereda E, Pedrolli C, Klersy C, Bonardi C, Quarleri L, Cappello S, et al. Nutritional status in older persons according to healthcare setting: a systematic review and meta-analysis of prevalence data using MNA. Clin Nutr. 2016;35(6):1282-90.

11. Davis JP, Wong AA, Schluter PJ, Henderson RD, O'Sullivan JD, Read SJ. Impact of premorbid undernutrition on outcome in stroke patients. Stroke. 2004;35(8):1930-4

12. Anker SD, John M, Pedersen PU, Raguso C, Cicoira M, Dardai E, et al. ESPEN guidelines on enteral nutrition: cardiology and pulmonology. Clin Nutr. 2006;25(2):311-8.

13. Bouillanne O, Morineau G, Dupont C, Coulombel I, Vincent JP, Nicolis I, et al. Geriatric nutritional risk index: a new index for evaluating at-risk elderly medical patients. Am J Clin Nutr. 2005;82(4):777-83.

14. Yamada K, Furuya R, Takita T, Maruyama Y, Yamaguchi Y, Ohkawa S, et al. Simplified nutritional screening tools for patients on maintenance hemodialysis. Am J Clin Nutr. 2008;87(1):106-13. 
15. Hsieh YW, Wang CH, Wu SC, Chen PC, Sheu CF, Hsieh CL. Establishing the minimal clinically important difference of the Barthel Index in stroke patients. Neurorehabil Neural Repair. 2007;21(3):233-8.

16. Sarmento LA, Pinto JS, da Silva AP, Cabral CM, Chiavegato LD. Effect of conventional physical therapy and Pilates in functionality, respiratory muscle strength and ability to exercise in hospitalized chronic renal patients: a randomized controlled trial. Clin Rehabil. 2017;31(4):508-20.

17. Inouye M, Kishi K, Ikeda Y, Takada M, Katoh J, Iwahashi M, et al. Prediction of functional outcome after stroke rehabilitation. Am J Phys Med Rehabil. 2000; 79(6):513-8.

18. Reeves MJ, Bushnell CD, Howard G, Gargano JW, Duncan PW, Lynch G, et al, Sex differences in stroke: epidemiology, clinical presentation, medical care, and outcomes. Lancet Neurol. 2008;7(10):915-26.

19. Granger CV, Dewis LS, Peters NC, Sherwood CC, Barrett JE. Stroke rehabilitation: analysis of repeated Barthel index measures. Arch Phys Med Rehabil. 1979;60(1):145-54.

20. Kobayashi I, Ishimura E, Kato Y, Okuno S, Yamamoto T, Yamakawa T, et al. Geriatric Nutritional Risk Index, a simplified nutritional screening index, is a significant predictor of mortality in chronic dialysis patients. Nephrol Dial Transplant. 2010;25(10):3361-5.

21. Beberashvili I, Azar A, Sinuani I, Shapiro G, Feldman L, Sandbank J, et al. Geriatric nutritional risk index, muscle function, quality of life and clinical outcome in hemodialysis patients. Clin Nutr. 2016;35(6):1522-9.

22. Yoshimura $Y$, Uchida $K$, Jeong $S$, Yamaga M. Effects of nutritional supplements on muscle mass and activities of daily living in elderly rehabilitation patients with decreased muscle mass: a randomized controlled trial. J Nutr Health Aging. 2016;20(2):185-91.

23. Dong J, Sundell MB, Pupim LB, Wu P, Shintani A, Ikizler TA. The effect of resistance exercise to augment long-term benefits of intradialytic oral nutritional supplementation in chronic hemodialysis patients. J Ren Nutr. 2011;21(2):149-59.

24. Martin-Alemañy G, Valdez-Ortiz R, Olvera-Soto G, Gomez-Guerrero I, Aguire-Esquivel G, Cantu-Quintanilla G, et al. The effects of resistance exercise and oral nutritional supplementation during hemodialysis on indicators of nutritional status and quality of life. Nephrol Dial Transplant. 2016;31(10):1712-20

25. Nii M, Maeda K, Wakabayashi H, Nishioka S, Tanaka A. Nutritional improvement and energy intake are associated with functional recovery in patients after cerebrovascular disorders. J Stroke Cerebrovasc Dis. 2016;25(1):57-62.

26. Nishioka S, Wakabayashi H, Nishioka E, Yoshida T, Mori N, Watanabe R. Nutritional improvement correlates with recovery of activities of daily living among malnourished elderly stroke patients in the convalescent stage: a cross-sectional study. J Acad Nutr Diet. 2016;116:837-43.

27. Nishioka S, Wakabayashi H, Momosaki R. Nutritional status changes and activities of daily living after hip fracture in convalescent rehabilitation wards: a retrospective observational cohort study from the Japan Rehabilitation Nutrition Database. J Acad Nutr Diet. 2018. https://doi.org/10. 1016/j.jand.2018.02.012

28. Uno C, Wakabayashi H, Maeda K, Nishioka S. Rehabilitation nutrition support for a hemodialysis patient with protein-energy wasting and sarcopenic dysphagia: a case report. Ren Replace Ther. 2018;4:18.

29. Alcorta MD, Alvarez PC, Cabetas RN, Martín MA, Valero M, Candela CG. The importance of serum albumin determination method to classify patients based on nutritional status. Clin Nutr ESPEN. 2018;25:110-3.

30. Maekawa M, Muramoto Y, Seimiya M, Kariyone K. Recommendation on measurement of serum albumin - how to handle the difference between measured values by BCG method and improved BCP method. Rinsho Byori. 2014;62(1):5-9 (in Japanese).

\section{Ready to submit your research? Choose BMC and benefit from:}

- fast, convenient online submission

- thorough peer review by experienced researchers in your field

- rapid publication on acceptance

- support for research data, including large and complex data types

- gold Open Access which fosters wider collaboration and increased citations

- maximum visibility for your research: over $100 \mathrm{M}$ website views per year

At $\mathrm{BMC}$, research is always in progress.

Learn more biomedcentral.com/submissions 